\title{
Comparing the diagnostic properties of skin scraping, adhesive tape, and dermoscopy in diagnosing scabies
}

\author{
Azmy A. Abdel-Latif ${ }^{1}$, Ahmad R. Elshahed ${ }^{1}$, Omar A. Salama $^{1}$, Mohamed L. Elsaie $^{2 \times}$
}

\begin{abstract}
Introduction: Scabies is a contagious skin infestation that mainly presents with itching at night and skin burrows that are visible to the naked eye. Diagnosing scabies with dermoscopy is still a matter of controversy. The aim of our study was to compare the diagnostic properties of adhesive tape, skin scraping, and dermoscopy in diagnosing scabies.

Methods: One hundred patients with clinical presumptive diagnoses of scabies underwent skin scraping, adhesive tape testing, and dermoscopic examination. Each diagnostic procedure was performed on three different areas.

Results: Comparing the diagnostic properties of the three methods, the adhesive tape test was the most sensitive method for diagnosing scabies. Sixteen cases (16.0\%) were definitely diagnosed as scabies using the adhesive tape test detecting the presence of mites or their eggs. Only 10 cases $(10.0 \%)$ were definitely diagnosed as scabies using the skin scraping test detecting mites or their eggs. Dermoscopic examination suggested a diagnosis of scabies in 22 cases (22.0\%), of which only 10 were definitely diagnosed as scabies by detecting mites using the adhesive tape test, skin scraping, or both.

Conclusion: The diagnosis of scabies can only be confirmed by seeing mites. The adhesive tape test and skin scraping procedure have high specificity in diagnosing scabies, but their low sensitivity cannot exclude the possibility of scabies. Dermoscopy-guided tape testing can be a helpful tool for better diagnosis of scabies.
\end{abstract}

Keywords: scabies, dermoscopy, adhesive tape

Received: 1 November 2017 | Returned for modification: 29 January 2018 | Accepted: 14 February 2018

\section{Introduction}

More than 300 million cases of scabies are reported annually worldwide. Severe itching that flares mostly at night and high contagiousness are the hallmark of scabies in humans (1). Diagnosing scabies is problematic and rests largely on patient history and clinical examination. In order to confirm a preliminary diagnosis of scabies, direct tests showing the presence of mites or their eggs by microscopic examination are a useful tool. Disadvantages encountered using such direct tests are a large margin of error and the time they require (2). Dermoscopy is viewed as a sensitive and valuable tool in diagnosing scabies in vivo. It permits the identification of a triangular or V-shaped structure corresponding to the front portion of the mite, including the head and legs. No wellcontrolled trials addressing accuracy in diagnosing scabies have been published despite many case reports signifying the potential usefulness of dermoscopy in scabies (3). No previously published research has assessed the accuracy of dermoscopy compared to skin scrapings or the use of adhesive tape in diagnosing scabies, which we aimed to evaluate.

\section{Patients and methods}

This study included 100 patients (6o males and 40 females) with a presumptive diagnosis of scabies. Their ages ranged from 3 months to 66 years. The diagnosis of scabies was made clinically using a case definition of established clinical parameters $(4,5)$. The presence of an itchy papular, crusted, or vesicular rash at certain predilection sites such as the interdigital spaces, flexion sites of the wrists, extension sites of the elbows, axillae, mamillae, perimammilar area, periumbicular area, penis, scrotum, or buttocks always raise suspicion of scabies (6). To fulfill a diagnosis of scabies, any individual must meet two of the following criteria: pruritus that intensifies at night, presence of lesions for $>2$ weeks, and at least one family member with similar lesions (7).

A severity score was calculated by adding the intensity of itching ( $0-4$ points), presence of superinfection ( $0-2$ points), and number of topographic areas affected (1-3 areas $=1$ point, 4-6 areas $=2$ points, $7-9$ areas $=3$ points, and $\geq 10$ areas $=4$ points). Hence, the severity score could range from o to 10 points (8); see Table 1.

Target areas were selected according to the symptoms, with more severely pruritic and symptomatic areas chosen for examination. Three different dermatologists carried out the three different procedures in accordance with their expertise. The procedures were carried out in the same order for all patients and the examining physician was blinded to the other methods' results. Table 1 | Assessing the severity of scabies.

\begin{tabular}{lcc}
\hline Symptom/sign & Intensity & Points \\
\hline Itching & None & 0 \\
& Mild & 1 \\
& Moderate & 2 \\
Topographic areas affected $(n)$ & Severe & 3 \\
& Associated sleep disturbances & 4 \\
& None & 0 \\
Presence of superinfection & $1-3$ & 1 \\
& $4-6$ & 2 \\
& $7-9$ & 3 \\
& $\geq 10$ & 4 \\
& No infection & 0 \\
& Pustules, suppuration, or abscess & 1 \\
& Constitutional symptoms & 2 \\
\hline
\end{tabular}




\section{Dermoscopy}

Dermoscopic identification of mites was performed at 10× magnification with a DermLite II Pro handheld dermoscope (3Gen, San Juan Capistrano, CA, USA). No liquid interface was required for application. The presence of a mite was suspected if a triangular structure with adjacent burrows was identified (Fig. 1). The dermoscope lenses were thoroughly cleaned with antiseptic cleansing wipes containing bactericidal and alcohol-based gel to minimize and inhibit any cross-contamination among patients.

\section{Skin scraping}

A hand magnifying lens was used to identify recent lesions suggestive of scabies infestation. Microscope immersion oil was applied to lesions or the scalpel blade and glass slides. Three different, non-excoriated, and non-inflamed areas were scraped with a scalpel blade and the scrapings were transferred from the three different sites to a single slide. A cover slip was placed over the slide. The entire slide was examined methodically under a low-power lens. If a mite was suspected, higher magnification was used.

\section{Adhesive tape test}

The adhesive tape test was performed as described by Katsumata and Katsumata (9). Transparent adhesive tape (3M Health Care) was used, which was cut into smaller strips the size of a microscope slide $(25 \times 50 \mathrm{~mm})$. These small strips were then firmly applied to the lesion and rapidly pulled off. The process was carried out in three different areas and the used tapes were moved to three slides. Slides were scanned at low-power magnification. If a mite was suspected, we used higher magnification.

\section{Follow-up}

All patients were treated with topical permethrin cream and reassessed after 2 weeks for improvement of their clinical condition using the severity score.

\section{Results}

Only 16 cases (16.0\%) were definitely diagnosed as scabies using the adhesive tape test to detect mites or their eggs. The adhesive tape test was negative in $84.0 \%$ of the cases studied. Ten cases $(10.0 \%)$ were definitely diagnosed as scabies using the skin scrap- ing test to detect mites or their eggs. One case was diagnosed as vagabond's disease by detection of a body louse. The other 89 cases were negative according to the skin scraping test. Dermoscopic examination of the patients studied suggested a diagnosis of scabies in only 22 cases (22.0\%). No dermoscopic findings suggested scabies in the other 78 cases (Table 2 ).

Table 2 | Distribution of the cases studied according to the different methods used.

\begin{tabular}{lc}
\hline Method and result & $\mathrm{n}(\%)$ \\
\hline Adhesive tape & \\
$\quad$ Negative & $84(84.0)$ \\
Positive & $16(16.0)$ \\
Skin Scraping & \\
$\quad$ Negative & $89(89.0)$ \\
Positive & $10(10.0)$ \\
$\quad$ Body louse & $1(1.0)$ \\
Dermoscope findings & \\
Negative & $78(78.0)$ \\
Suggestive positive & $22(22.0)$ \\
Certain positive & $10(45.5)$ \\
Not sure positive & $12(54.5)$ \\
\hline
\end{tabular}

Of the 22 cases dermoscopically suggestive of scabies, only 10 were definitely diagnosed as scabies by detection of mites using the adhesive tape test, skin scraping, or both (Figs. 2-4). The other 12 cases dermoscopically suggestive of scabies were considered negative for scabies because mites or their eggs were not detected by the adhesive tape test or skin scraping.

Excoriations were the most common dermoscopic findings because they were found in 90 cases $(90.0 \%)$. However, they were not considered suggestive findings for scabies. The triangle sign, which consists of the "head" portion of the mite and the first two pairs of legs, was found in 16 cases $(16.0 \%)$ and a burrow was found in only nine cases $(9.0 \%)$. Both were considered suggestive findings for scabies. Microvesiculations were found in only five cases (5.0\%). The sensitivity of the adhesive tape test was higher than that of dermoscopy and skin scraping. By definition, the specificity and the positive predictive value of the adhesive tape test and skin scraping were each 100\%. Dermoscopy and skin scraping have the same sensitivity (Table 3 ).

Table 3 | Diagnostic properties of diagnostic procedures for scabies.

\begin{tabular}{lccc}
\hline Diagnostic property & Dermoscopy & $\begin{array}{c}\text { Adhesive } \\
\text { tape test }\end{array}$ & $\begin{array}{c}\text { Skin } \\
\text { scraping }\end{array}$ \\
\hline Sensitivity (TP/TP+FN) & $43.47 \%$ & $69.56 \%$ & $43.47 \%$ \\
Specificity (TN/TN+FP) & $84.41 \%$ & $100 \%$ & $100 \%$ \\
Negative predictive value (TN/TN+FN) & $83.33 \%$ & $91.66 \%$ & $85.55 \%$ \\
Positive predictive value (TP/TP+FP) & $45.45 \%$ & $100 \%$ & $100 \%$ \\
\hline
\end{tabular}

$\mathrm{FN}=$ false negative, $\mathrm{TN}=$ true negative, $\mathrm{TP}=$ true positive, $\mathrm{FP}=$ false positive.

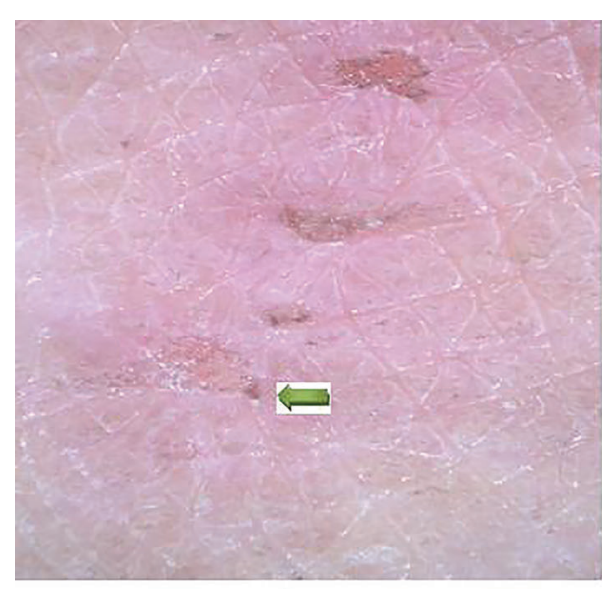

$A$

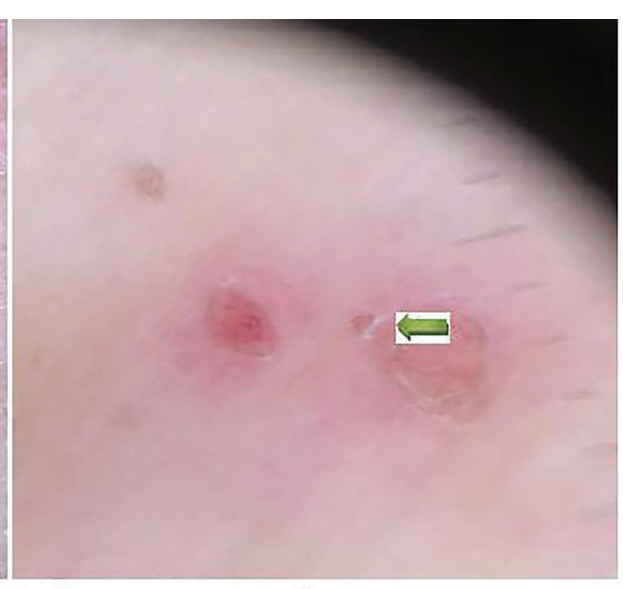

B

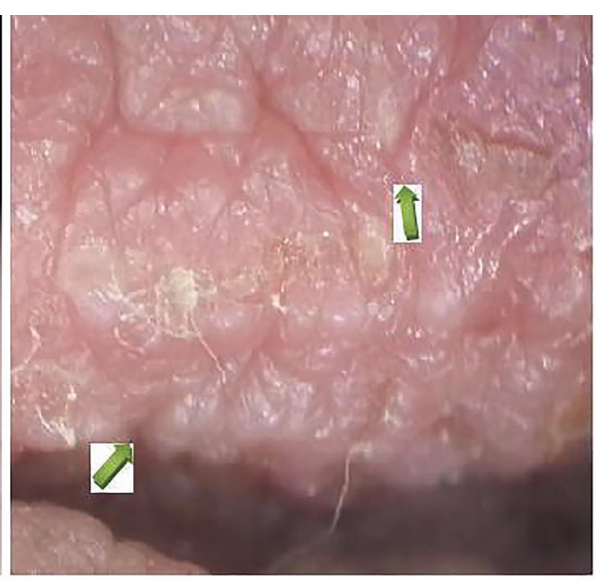

$\mathrm{C}$

Figure 1 ( Positive dermoscopic signs. A) and B) triangle signs; C) burrow detected on the glans penis. 


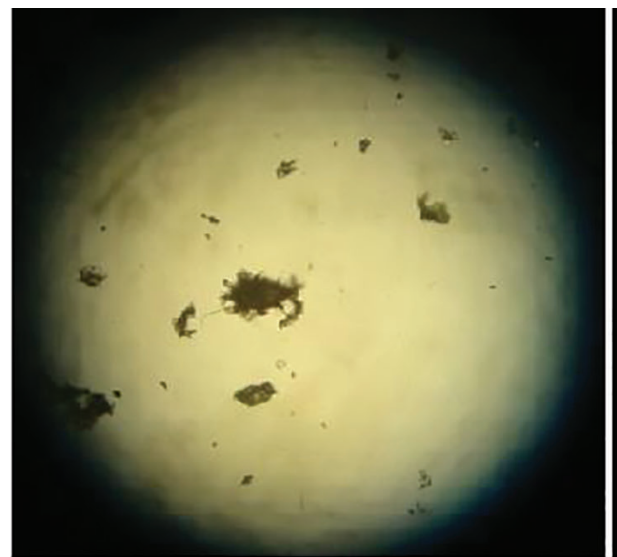

A

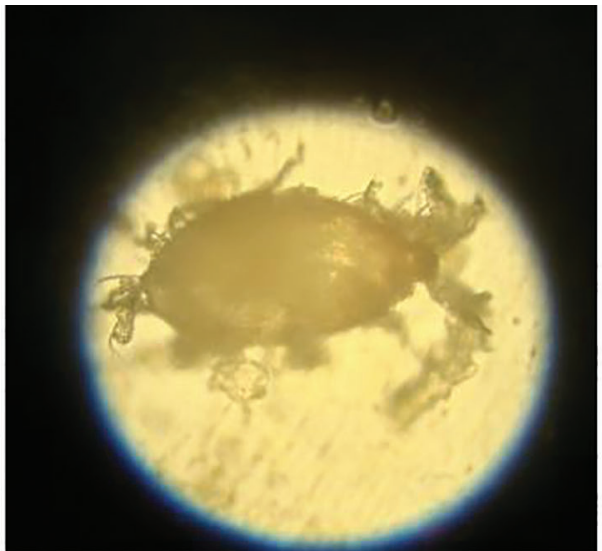

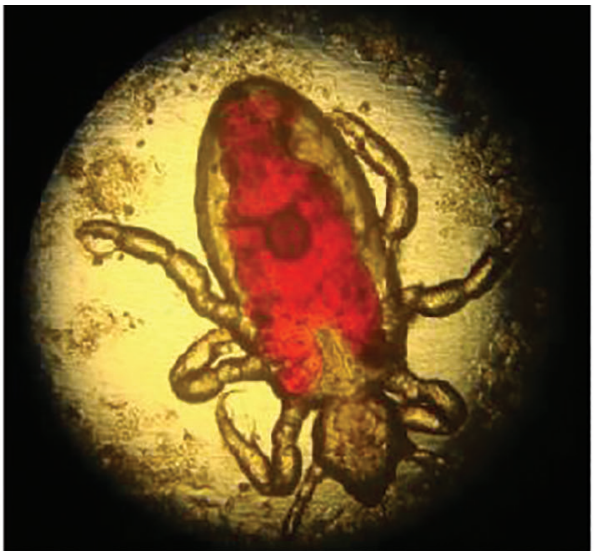

Figure 2 | Skin scraping results. A) adult mite, low-power magnification; B) adult mite, high-power magnification; C) adult body louse in a case of vagabond's disease.

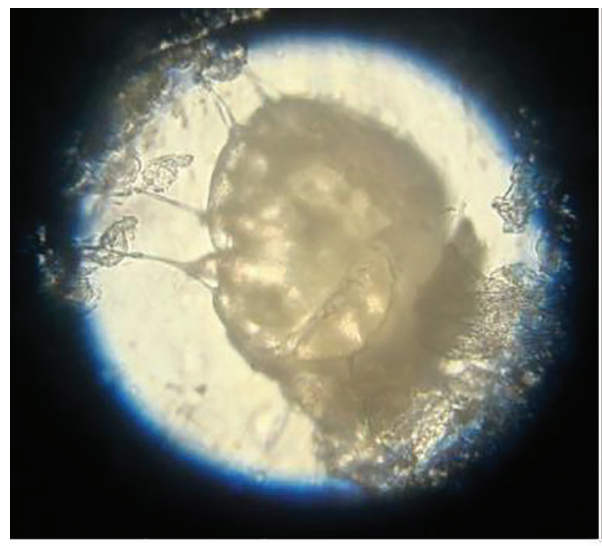

$A$

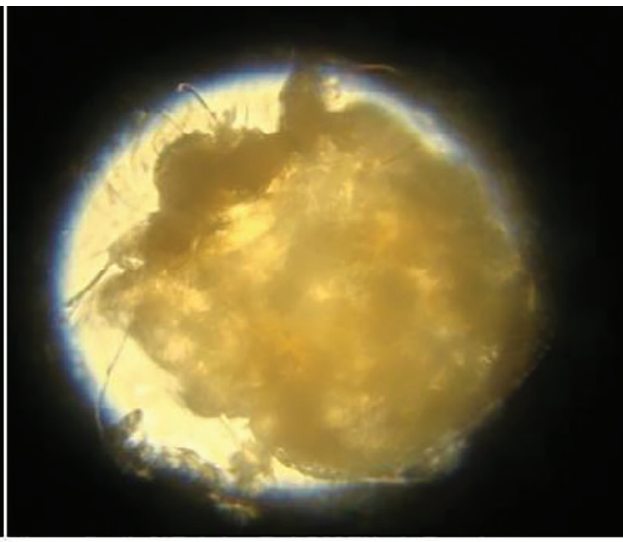

B

Figure 3 | Adhesive tape test results. A) adult mite, 40x; B) adult mite, high-power magnification.

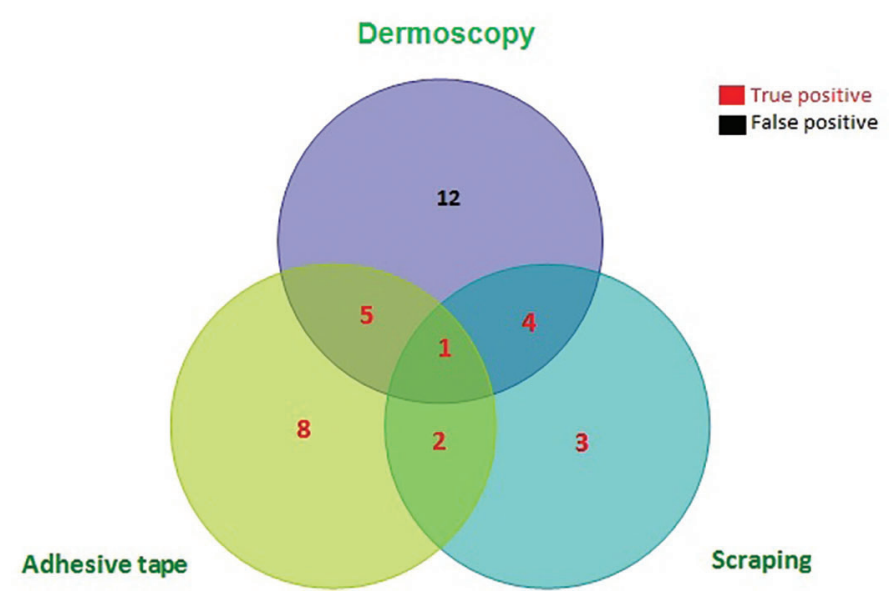

Figure 4 | Overlap between positive findings in different study modalities.

\section{Discussion}

Diagnosing scabies is challenging and important. More than 300 million cases are diagnosed annually worldwide, with a greater prevalence in developing countries (10).

In countries with a low socioeconomic status, clinical identification of scabies is the main diagnostic tool. These clinical criteria are prone to error due to the similarity of scabies with other infectious and noninfectious diseases. A definitive diagnosis can only be made through identification of mites, eggs, or mite pellets using ex vivo microscopic examination (11).

Apart from ex vivo examination, identification can be per- formed using a simpler noninvasive technique originally known as epiluminescence microscopy. The front portion of the mite can be visualized with this procedure and it may provide a helpful clue towards a more accurate diagnosis of scabies (12). Even though mites must be seen in order to diagnose scabies, not seeing a mite cannot exclude the presence of scabies. Many cases with no mites seen were misdiagnosed as eczema, and were confirmed as scabies during further return consultations once mites were identified (13).

Only 23 cases $(23.0 \%)$ were definitely diagnosed as scabies by detection of mites or their eggs using the adhesive tape test, skin scraping, or both, compared to $36.3 \%$ and $52.0 \%$ in two previous studies $(5,14)$; see Table 4 . Nevertheless, our findings were compatible with those of Palicka et al., who showed that in 151 patients in whom skin scrapings were positive only $18.0 \%$ of the samples contained mites (15). Seventy-seven (77.0\%) of the cases were not diagnosed as scabies, and one was diagnosed as vagabond's disease.

The adhesive tape test was the most sensitive method when compared with the scraping test technique and dermoscopy. Its sensitivity was $69.6 \%$ and only slightly higher than that determined in the study by Walter et al. (68.0\%). The adhesive tape test relies on disrupting the stratum corneum along with the epidermis and the attached mites. The mites then stick to the tape on the stretched skin (6).

The sensitivity of the adhesive tape test $(69.6 \%)$ was higher than that of the skin scraping test $(43.5 \%)$, which was considered the reference procedure for scabies diagnosis for decades. These findings are also compatible with those of Walter et al., who re- 
Table 4 | Results of diagnostic procedures for scabies in 100 patients and their interpretation.

\begin{tabular}{|c|c|c|c|c|c|}
\hline Skin scraping & Adhesive tape test & Dermoscopy & Patients $(n)$ & Final diagnosis of scabies & Interpretation \\
\hline Positive & Positive & Positive & 1 & Yes & TP scraping/TP tape test/TP dermoscopy \\
\hline Positive & Positive & Negative & 2 & Yes & TP scraping/TP tape test/FN dermoscopy \\
\hline Positive & Negative & Positive & 4 & Yes & TP scraping/FN tape test/TP dermoscopy \\
\hline Positive & Negative & Negative & 3 & Yes & TP scraping/FN tape test/FN dermoscopy \\
\hline Negative & Positive & Positive & 5 & Yes & FN scraping/TP tape test/TP dermoscopy \\
\hline Negative & Positive & Negative & 8 & Yes & FN scraping/TP tape test/FN dermoscopy \\
\hline Negative & Negative & Positive & 12 & No & TN scraping/TN tape test/FP dermoscopy \\
\hline Negative & Negative & Negative & 65 & No & TN scraping/TN tape test/TN dermoscopy \\
\hline
\end{tabular}

$\mathrm{FN}=$ false-negative, $\mathrm{FP}=$ false-positive, $\mathrm{TN}=$ true-negative, $\mathrm{TP}=$ true-positive.

ported that the sensitivity of skin scraping is about $46.0 \%$. This can be explained by the larger amount of debris in the scraping compared to that in the adhesive tape, which would obscure the identification and visualization of the mites (5).

The sensitivity of dermoscopy was (43.5\%), which is very low when compared with that of Walter et al. (83.0\%) and Dupuy et al. $(91.0 \%)(8,14)$. It is possible that the pigmented skin types of our patients might have affected and lowered the sensitivity of the test. Dark pigments of the skin might have interfered with visualizing the delta wing sign corresponding to the brownish-black anterior part of the mites (16). Moreover, any dirt, artifacts on slides, small crusts, or even tiny bleeding spots could have interfered with misdiagnosing a mite.

The specificity of the dermoscope was very high $(84.4 \%)$ if compared with the study by Walter et al., who reported that the specificity of dermoscopy is about $46.0 \%$. However, it is slightly lower than in the study by Dupuy et al., who reported that the specificity of dermoscopy is about $86.0 \%(8,14)$. The high specificity of dermoscopy in scabies diagnosis was not actually due to the dermoscopic results but mainly due to the small number of cases that were definitely diagnosed as scabies (Table 5).

Table 5 | Comparison of sensitivity and specificity of using dermoscopes to diagnose scabies with other studies.

\begin{tabular}{lccc}
\hline Variable & \multicolumn{3}{c}{ Study (Reference) } \\
Sensitivity & $43.5 \%$ & Walter et al. (8) & Dupuy et al. (14) \\
Specificity & $84.4 \%$ & $83.0 \%$ & $91.0 \%$ \\
\hline
\end{tabular}

The adhesive tape test and skin scraping procedure have high specificity in diagnosing scabies, but their low sensitivity cannot exclude the possibility of scabies. An important aspect that is considered a disadvantage by many when using a dermoscope is the requirement of good training to avoid confusion of artifacts. Moreover, it is more of a research tool than an established diagnostic method for identifying and diagnosing scabies. In view of these results, a sensitive and specific method for diagnosing scabies is still lacking. We suggest that dermoscopy-guided tape testing in diagnosing scabies may be helpful and should be studied.

\section{References}

1. Micali G, Lacarrubba F, Verzì AE, Chosidow 0 , Schwartz RA. Scabies: advances in noninvasive diagnosis. PLoS Negl Trop Dis. 2016;10:e0004691.

2. Lacarrubba F, Verzì AE, Dinotta F, Scavo S, Micali G. Dermatoscopy in inflammatory and infectious skin disorders. G Ital Dermatol Venereol. 2015;150:521-31.

3. Errichetti E, Stinco $G$. The practical usefulness of dermoscopy in general dermatology. G Ital Dermatol Venereol. 2015;150:533-46.

4. Hassan HA, Ezzat W, Lebshtein A. Scabies as a health problem among primary school children in Cairo. J Egypt Public Health Assoc. 1979;54:65-75.

5. Heukelbach J, Walton SF, Feldmeier H. Ectoparasitic infestations. Curr Infect Dis Rep. 2005;7:373-80.

6. McCarthy JS, Kemp DJ, Walton SF, Currie BJ. Scabies: more than just an irritation. Postgrad Med J. 2004;80:382-7.

7. Jackson A, Heukelbach J, Filho AF, Júnior Ede B, Feldmeier H. Clinical features and associated morbidity of scabies in a rural community in Alagoas, Brazil. Trop Med Int Health. 2007;12:493-502.

8. Walter B, Heukelbach J, Fengler G, Worth C, Hengge U, Feldmeier H. Comparison of dermoscopy, skin scraping, and the adhesive tape test for the diagnosis of scabies in a resource-poor setting. Arch Dermatol. 2011;147:468-73.

9. Katsumata K, Katsumata K. Simple method of detecting Sarcoptes scabiei var hominis mites among bedridden elderly patients suffering from severe scabies infestation using an adhesive tape. Intern Med. 2006;45:857-9.
10. Currier RW, Walton SF, Currie BJ. Scabies in animals and humans: history, evolutionary perspectives, and modern clinical management Ann N Y Acad Sci. 2011;1230: E50-60.

11. Chosidow O. Clinical practice. Scabies. N Engl J Med. 2006;354:1718-27.

12. Prins C, Stucki L, French L, Saurat JH, Braun RP. Dermoscopy for the in vivo detection of Sarcoptes scabiei. Dermatology. 2004;208:241-3.

13. Albrecht J, Bigby M. Testing a test: critical appraisal of tests for diagnosing scabies. Arch Dermatol. 2011;147:494-7.

14. Dupuy A, Dehen L, Bourrat E, Lacroix C, Benderdouche M, Dubertret L, et al. Accuracy of standard dermoscopy for diagnosing scabies. J Am Acad Dermatol. 2007;56:53-62.

15. Palicka P, Malis L, Samsinák K, Zitek K, Vobrázková E. Laboratory diagnosis of scabies. J Hyg Epidemiol Microbiol Immunol. 1980;24:63-70.

16. Feldmeier H. Diagnosis of parasitic skin diseases. In: Maibach H, Gorouhi F, editors. Evidence-based dermatology. 2nd ed. New York: McGraw-Hill Medical; 2010. p. 73-86. 